\title{
MANTISPIDAE (NEUROPTERA) DO MUSEU DE ZOOLOGIA DA UEFS
}

\author{
Hémille Mariane Dias Oliveira'; Freddy Ruben Bravo Quijano² \\ 1. Bolsista PIBIC/CNPq, Graduando em Bacharelado em Ciências Biológicas, Universidade Estadual de Feira de Santana, e- \\ mail: hemille.dias@hotmail.com \\ 2. Orientador, Departamento de Ciências Biológicas, Universidade Estadual de Feira de Santana, e-mail: fbravo@uefs.br
}

PALAVRAS-CHAVE: Riqueza, Semiárido, Levantamento.

\section{INTRODUÇÃO}

Mantispidae Leach, 1815 é uma das 17 famílias da ordem Neuroptera, subordem Hemerobiiformia. Os insetos desta família assemelham-se a louva-a-deuses (Mantodea), com pernas anteriores, raptoriais, e protórax alongado (figura 1) (Freitas \& Penny, 2012). Os mantispídeos são holometábolos e predadores em todas as fases do desenvolvimento, inclusive da larval onde costuma predar ovissacos de aranha e ninhos de vespas aculeadas (Hoffman, 2002).

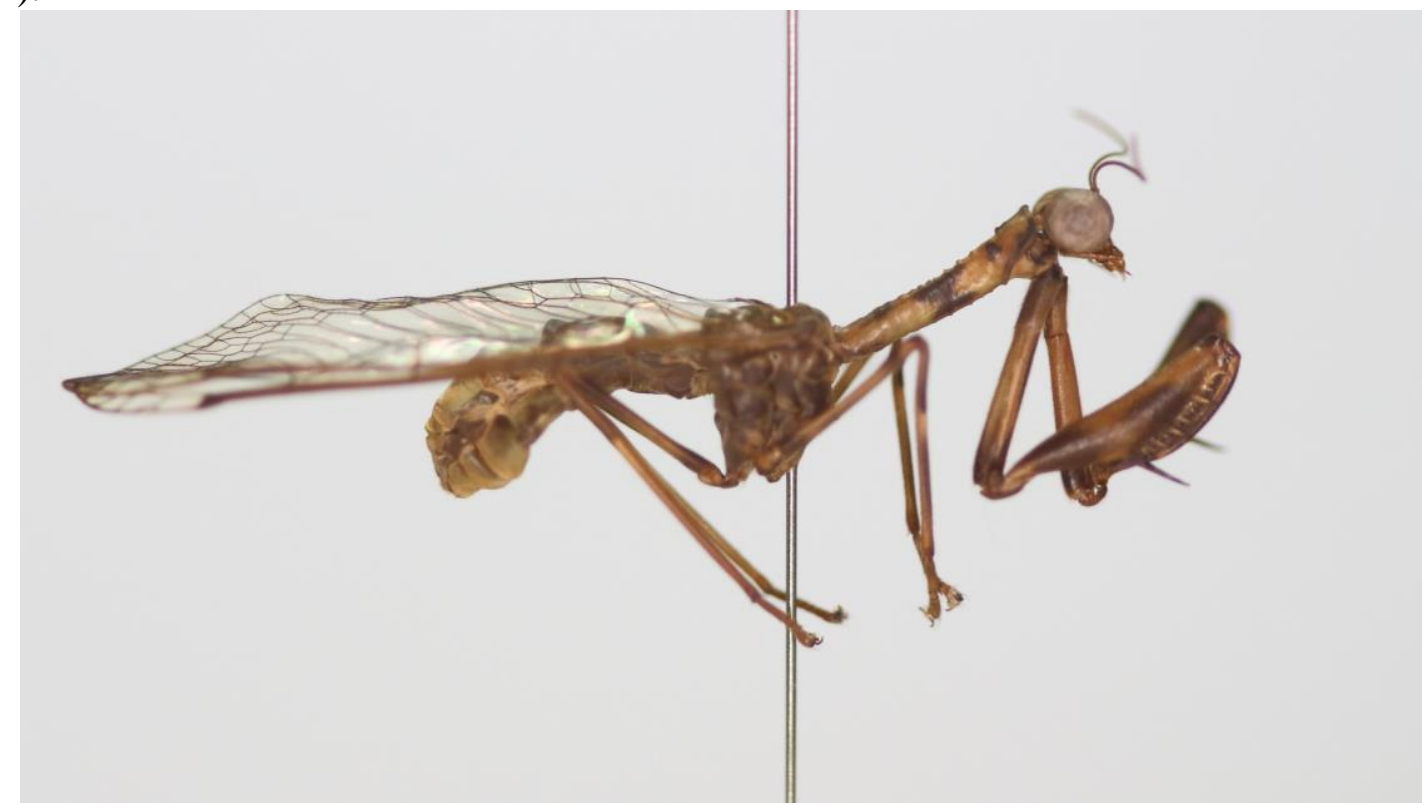

Figura 1. Buyda phitisica (hábito)

A família Mantispidae é considerada cosmopolita, ausente apenas na Antártida e é classificada nas subfamilias Calomantispinae, Symphrasinae, Drepanicinae e Mantispinae. No Brasil há registro apenas das últimas três. Nas Américas são conhecidos 44 gêneros e 410 espécies e subespécies (Camacho \& García, 2015), e para o Brasil são registrados 13 gêneros e 55 espécies (Machado \& Martins, 2016).

O Nordeste e o semiárido brasileiros tem sido historicamente negligenciados para estudos sobre a fauna de insetos e com Mantispidae não tem sido diferente, portanto dados sobre riqueza de Mantispidae para estas regiões são escassos. A coleção de insetos do Museu de Zoologia da UEFS conta com exemplares de Mantispidae do Brasil, inclusive das regiões Nordeste e Semiárido que foram e continuam sendo estudados, assim, esta pesquisa vem a contribuir para sanar esta deficiência de informação e conhecimento por meio da produção de uma lista de espécies, incluindo espécies novas e novos registros para o semiárido, direcionando a produção científica e publicação de trabalhos acadêmicos. 


\section{MATERIAL E MÉTODOS}

A Coleção Professor Johann Becker do Museu de Zoologia da Universidade Estadual de Feira de Santana (MZFS) conta com espécimes de Mantispidae de várias localidades do país e principalmente do Semiárido brasileiro montados em alfinete entomológico e em álcool 70\%. Os espécimes em álcool 70\% foram montados em alfinete entomológico com a asas do lado direito abertas para melhor visualização. Foram secos em estufa a $42{ }^{\circ} \mathrm{C}$ por pelo menos 10 dias. Após esse período, foram etiquetados e receberão um número de tombo do Museu.

Para a identificação dos espécimes, foi retirada a parte apical do abdômen e colocadas em ácido lático $85 \%$ quente por até 30 minutos para clareamento das partes esclerotizadas. As terminálias foram examinadas sob lupa e microscópio em glicerina (Machado \& Rafael, 2010). Foram realizados fotos e desenhos da cabeça, asas e terminálias masculina e feminina. Para a identificação das espécies foram utilizadas as chaves de identificação presentes nos trabalhos de Camacho \& García (2015); Machado \& Rafael (2010); Penny \& da Costa (1983).

\section{RESULTADOS E DISCUSSÃO}

O MZFS conta com espécimes pertencentes a apenas duas subfamílias: Mantispinae e Symphrasinae. O material previamente montado e tombado no MZFS apresenta espécimes de um gênero e uma espécie da subfamília Symphrasinae e cinco gêneros e sete espécies da subfamília Mantispinae. A subfamília Drepanicinae não se encontra na coleção MZFS.

No MZFS há três gêneros e três espécies da subfamília Symphrasinae e seis gêneros e nove espécies da subfamília Mantispinae. O material apresenta registros de Roraima, Pará, Ceará, Rio Grande do Norte, Sergipe, Bahia, Minas Gerais, Goiás, Rio de Janeiro, Santa Catarina e Rio Grande do Sul. Duas espécies são registradas pela primeira vez para o Semiárido, uma com gênero encontrado pela primeira vez no semiárido e outra considerado como expansão do território para o Norte, assim como, também, uma espécie nova do gênero Trichoscelia (figura 2) ainda não publicada.

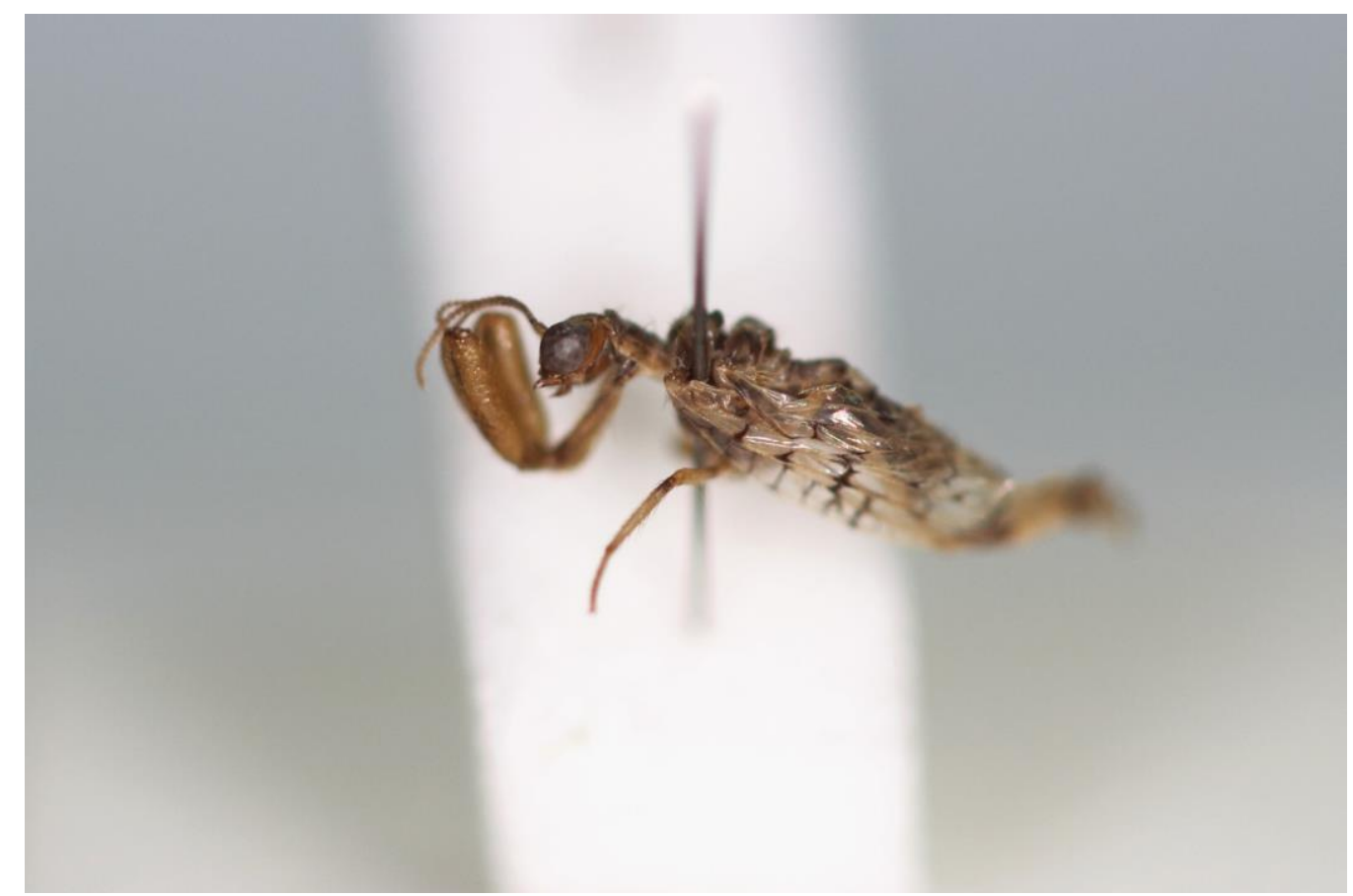

Figura 2. Trichoscelia sp. n. 
Houve também novos registros para a Bahia, Rio Grande do Norte, Ceará e Sergipe para quatro espécies que já apresentavam registro no Semiárido brasileiro. Uma foi registrada pela primeira vez para Feira de Santana; vale ressaltar que apenas um espécime foi coletado durante a execução do projeto.

A nova espécie Trichoscelia sp. n. tem como característica diagnose a disposição dos três espinhos apicais do gonocoxito (figura 3). Trichoscelia sp. n. apresenta características semelhantes a espécie Tricoscelia sequella encontradas na chave de identificação em Penny \& da Costa (1983). Porém se diferem pela forma e quantidade de flagelômeros, pelas células fechadas na asa anterior, posteriores à base da primeira célula radial (figura 4) e pela disposição dos espinhos do gonocoxito

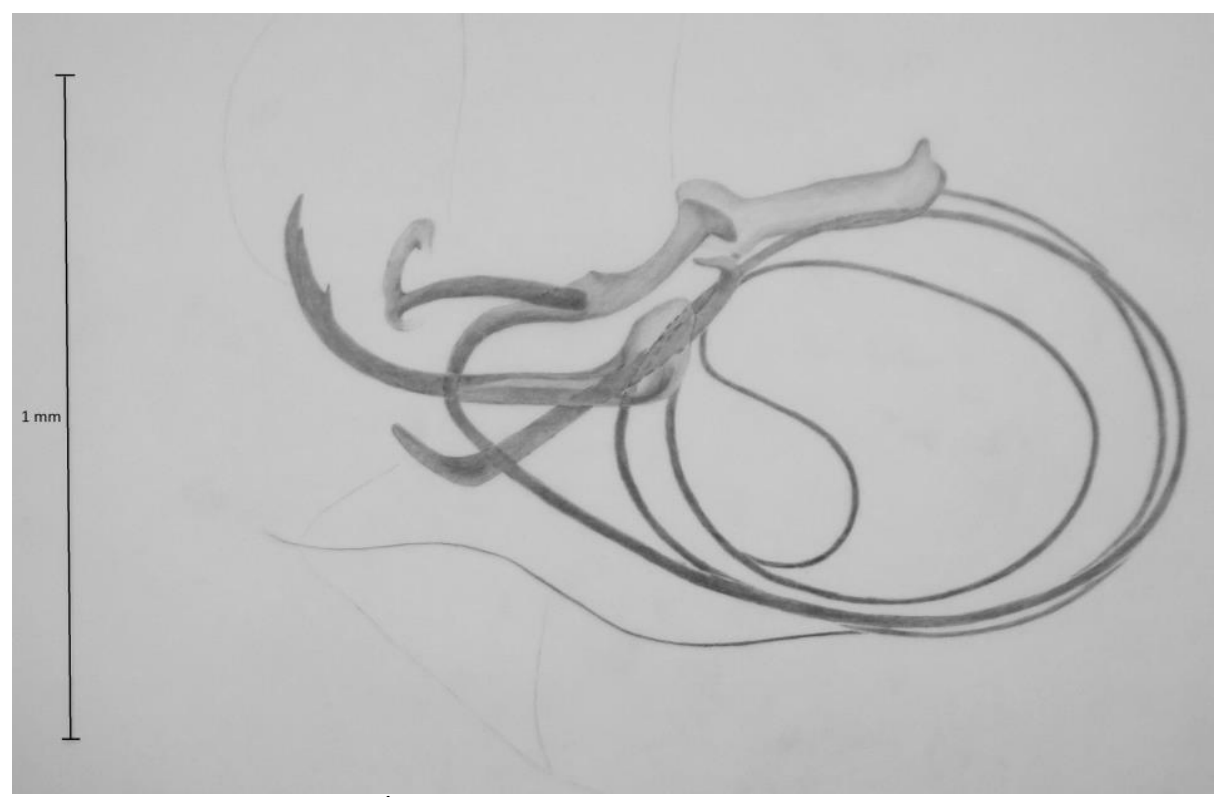

Figura 3. Ápice do abdome de Trichoscelia sp. n.

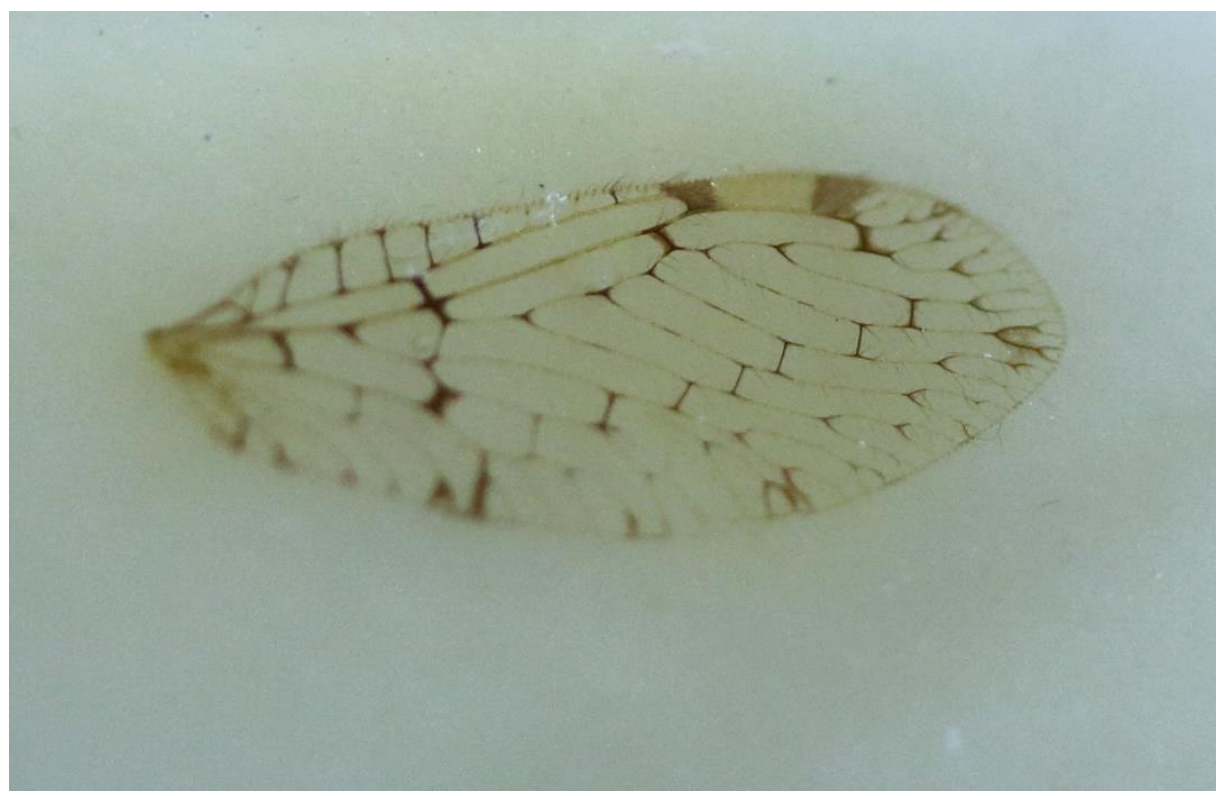

Figura 4. Asa anterior de Trichoscelia sp. n. 
O MZFS conta com 2 subfamílias, 9 gêneros e 12 espécies, de diversos estados brasileiros. Anteriormente tinham conhecimento de apenas seis espécies com registro para o semiárido brasileiro, apresentando agora nove espécies registradas, onde uma das 3 com novo registro é uma espécie nova. Os registros continuam sendo para apenas duas subfamílias, das três presentes no Brasil.

\section{REFERÊNCIAS}

Ardila-Camacho, A. \& García, A. 2015. Mantid $\square$ ies of Colombia (Neuroptera, Mantispidae). Zootaxa, 3937(3): 401-455.

Freitas, S. \& Penny, N. 2012. Neuroptera. p. 538-546. In: J. A. Rafael, G.A.R. Melo, C.J.B., Carvalho, S.A. Casari \& Constantino, R (Orgs). Insetos do Brasil: Diversidade e Taxonomia, São Paulo, Holos Editora, 810p.

Grimaldi, D. \& Engel, M. 2005. Evolution of the Insects. New York, Cambridge University Press, p. 755.

Hoffman, K.M. (2002) Family Mantispidae. In Penny, N. D. (ed.), A guide to the lacewings (Neuroptera) of Costa Rica. Proceedings of the California Academy of Sciences, 53 (12), 251275.

Machado, R. \& Rafael, J. 2010. Taxonomy of the Brazilian species previouslu placed in Mantispa Illiger, 1798 (Neuroptera: Mantispidae), wich the description of three new species. Zootaxa, 2454: 1-61.

Machado, R.J.P, \& Martins, C.C 2016. Mantispidae. In: Catálogo Taxonômico da Fauna do Brasil. PNUD. Disponível em: http://fauna.jbrj.gov.br/fauna/faunadobrasil/1694. Acesso em: 06 Out. 2016

Machado RJP,Martins CC 2017. Mantispidae In: Catálogo Taxonômico da Fauna do Brasil. PNUD. Disponível em: <http://fauna.jbrj.gov.br/fauna/faunadobrasil/3213 >. Acesso em: 28 Jul. 2017

Penny, N.D. (1982a) Review of the generic level classification of New World Mantispidae (Neuroptera). Acta Amazonica, 12 (1), 209-223

Penny, N.D. (1982b) Neuroptera of the Amazon basin. Part 6. Mantispidae (1). Acta Amazônica, 12 (2), 415-463.

Penny, N. D. \& da Costa, C. A. 1983. Mantispídeos do Brasil (Neuroptera: Mantispidae). Acta Amazonica, 13(3-4): 601-687.

Redborg, K. E. 1998. Biology of the Mantispidae. Annual Review of Entomolology, 43:17594. 